\title{
Erratum to: 'TElmisartan in the management of abDominal aortic aneurYsm (TEDY): The study protocol for a randomized controlled trial'
}

\author{
Dylan R. Morris ${ }^{1 \dagger}$, Margaret A. Cunningham ${ }^{2 \dagger}$, Anna A. Ahimastos ${ }^{3 \dagger}$, Bronwyn A. Kingwell $^{3}$, Elise Pappas ${ }^{1}$, \\ Michael Bourke ${ }^{4}$, Christopher M. Reid ${ }^{5}$, Theo Stijnen ${ }^{6}$, Ronald L. Dalman ${ }^{7}$, Oliver O. Aalami ${ }^{7}$, Jan H. Lindeman ${ }^{6}$, \\ Paul E. Norman ${ }^{8}$, Philip J. Walker ${ }^{9}$, Robert Fitridge ${ }^{10}$, Bernie Bourke ${ }^{4}$, Anthony E. Dear ${ }^{11}$, Jenna Pinchbeck ${ }^{1}$, \\ Rene Jaeggi ${ }^{1}$ and Jonathan Golledge ${ }^{1,12^{*}+}$
}

Unfortunately, the original version of this article ${ }^{1}[1]$ contained an error. Professor Jonathan Golledge is incorrectly referred to as deceased in the published article.

\section{Endnote}

${ }^{1}$ Dylan R. Morris, Margaret A. Cunningham, Anna A. Ahimastos, Bronwyn A. Kingwell, Elise Pappas, Michael Bourke, Christopher M. Reid, Theo Stijnen, Ronald L. Dalman, Oliver O. Aalami, Jan H. Lindeman, Paul E. Norman, Philip J. Walker, Robert Fitridge, Bernie Bourke, Anthony E. Dear, Jenna Pinchbeck1, Rene Jaeggi and Jonathan Golledge: TElmisartan in the management of abDominal aortic aneurYsm (TEDY): The study protocol for a randomized controlled trial. Trials 2015, 16:274

\section{Author details}

${ }^{1}$ Queensland Research Centre for Peripheral Vascular Disease, School of Medicine and Dentistry, James Cook University, Townsville, QLD, Australia. ${ }^{2}$ Psychology Department, University of Stirling, Stirling, UK. ${ }^{3}$ Baker IDI Heart and Diabetes Institute and The Department of Cardiovascular Medicine, Alfred Hospital Melbourne, Melbourne, Australia. ${ }^{4}$ Gosford Vascular Services, Gosford, New South Wales, Australia. ${ }^{5}$ Centre of Cardiovascular Research and Education in Therapeutics, Department of Epidemiology and Preventive Medicine, Monash University, Alfred Hospital, Melbourne, Australia. ${ }^{6}$ Leiden University Medical Center, Leiden, The Netherlands. 'Division of Vascular Surgery, Department of Surgery, Stanford University School of Medicine, Stanford, CA, USA. ${ }^{8}$ School of Surgery, University of Western Australia, Perth, WA, Australia. ${ }^{9}$ University of Queensland School of Medicine, Discipline of Surgery and Centre for Clinical Research, and Department of Vascular

\footnotetext{
* Correspondence: jonathan.golledge@jcu.edu.au

${ }^{\dagger}$ Equal contributors

${ }^{1}$ Queensland Research Centre for Peripheral Vascular Disease, School of Medicine and Dentistry, James Cook University, Townsville, QLD, Australia

${ }^{12}$ The Department of Vascular and Endovascular Surgery, The Townsville Hospital, Townsville, QLD, Australia

Full list of author information is available at the end of the article
}

Surgery, Royal Brisbane and Women's Hospital, Brisbane, Queensland, Australia. ${ }^{10}$ Department of Surgery, University of Adelaide, The Queen Elizabeth Hospital, Adelaide, South Australia, Australia. ${ }^{11}$ Eastern Health Clinical School, Department of Medicine, Monash University, Melbourne, Australia. ${ }^{12}$ The Department of Vascular and Endovascular Surgery, The Townsville Hospital, Townsville, QLD, Australia.

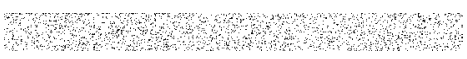

\section{Reference}

1. Morris DR, Cunningham MA, Ahimastos AA, Kingwell BA, Elise P, Michael B, et al. TElmisartan in the management of abDominal aortic aneurYsm (TEDY): The study protocol for a randomized controlled trial. Trials. 2015;16:274.
Submit your next manuscript to BioMed Central and we will help you at every step:

- We accept pre-submission inquiries

- Our selector tool helps you to find the most relevant journal

- We provide round the clock customer support

- Convenient online submission

- Thorough peer review

- Inclusion in PubMed and all major indexing services

- Maximum visibility for your research

Submit your manuscript at www.biomedcentral.com/submit 